\title{
The Status and Psychological Challenges of Implementation of Continuous Professional Development/CPD/ in Primary Schools of South Wollo Zone
}

\section{Tareke $\mathbf{G}^{1 *}$ and Tefera $\mathbf{B}^{2}$}

${ }^{1}$ Tareke G, Wollo University, Dessie, Ethiopia

${ }^{2}$ Tefera B, Addis Ababa University, Ethiopia

\begin{abstract}
The purpose of this study was to assess the effectiveness of Continuous Professional Development (CPD) implementation and to better understand the relationship between teachers' outlook and CPD implementation in primary schools of South Wollo zone. A total of 90 first and 96 second cycle primary teachers, 24 principals and vice principals, 11 supervisors, 4 education experts from sample woredas and two experts from the zone education office participated in the study. To collect data about the practice of CPD at school level and its effect on classroom teaching questionnaires were administered to teachers, principals, vice principals and supervisors. In addition, a questionnaire was administered to teachers to generate data regarding teachers' perception of CPD. Pertaining to teachers self-efficacy, scales were adapted from Tschannen-Moran and Woolfok Hoy. To secure qualitative data, interview, class session observation and document analysis were used. The finding of the study indicated that first cycle primary school teachers had higher level of participation in CPD learning activities than second cycle primary school teachers. Most of the CPD activities were confined to group discussion in second cycle primary schools. Teachers involved in the study were confined largely to traditional methods of presenting classroom lesson. The result also indicated that first cycle primary school teachers and newly qualified teachers have optimistic perception towards teachers' professional development activities. Finally it was found that teachers with low self-efficacy tend to show better commitment and motivation to participate in CPD training. Generally, the objectives of the CPD program were not achieved. Given the generally insufficient implementation of CPD in the site of the study, it is suggested that more effort be made to inculcate among teachers the importance of the program and provide teachers with greater support to help them perform this duty.
\end{abstract}

Keywords: Continuous professional development; Perception; Selfefficacy; Newly deployed teachers; Experienced teachers

\section{Introduction}

Among the most important factors that determine quality in education in general and teaching learning process in particular are teachers' competencies [1,2]. Darling-Hammond said that the skills and knowledge of teachers outweigh other factors to influence student learning. Supporting this $[3,4]$ also pointed that the core determining factor of student achievement is the skills and knowledge possessed by teachers.

Many writers consider the pre-service training as the only preparation teachers receive during their career. However, scholars in the field of teacher education argue that the knowledge, skills and attitude needed for quality teaching could not fully develop during the pre-service teacher education alone [4,5]. For instance, Leu [4] concludes that the pre-service training produces neither the teaching skill nor the knowledge and attitude required for improving classroom approach and student learning [6]. Also observe that teachers selfconfidence and their perception about their ability to make difference in student achievement determines the successful implementation of professional activities in the classroom. Recently local studies have been conducted on the practice of teachers' CPD program. For instance, [7-11] examined the practice of CPD activities both in primary and secondary schools. The results of these studies have shown that there have been several factors impeding the implementation of the CPD program. According to these studies, insufficient planning, organization and monitoring of the program, scarcity of resources to sustain the program; too much reliance on external and one- shot training and lack of knowledge of the school administrative body are the main constraints hindering teachers' CPD program considerably.

However, the aforementioned studies gave very little attention to teachers' characteristics such as their self-efficacy belief and perception of CPD. Accordingly there is a pressing need for continuous learning for updating teacher's skills, knowledge and classroom practice. Bearing this in mind the Ministry of Education in collaboration with donor agencies has launched a new scheme of teachers' continuous professional development /CPD/program. To implement the CPD program in 1909 the Ministry of Education produced a CPD implementation guideline which serves as the working document to practice CPD strategy. The framework entails that individual teachers are self-reflective learners who strive to gain new knowledge, improve their skill through private reading, learn from peers, talented colleagues, and reflect on their own classroom practice and conducting action research. This implies that teachers can take responsibilities for planning and enacting developmental activities for updating their prior knowledge and skills. Thus assessing teachers' perception and their efficacy believe is timely and important in this regard.

\section{Research Methodology}

\section{Population}

The participants of the study were primary school teachers. In

*Corresponding author: Tareke G, Wollo University, Dessie, Ethiopia, Tel: 033311-5204; 033-311-5247; E-mail: girumtareke@yahoo.com

Received December 13, 2017; Accepted May 30, 2018; Published June 04, 2018

Citation: Tareke G, Tefera B (2018) The Status and Psychological Challenges of Implementation of Continuous Professional Development/CPD/ in Primary Schools of South Wollo Zone. J Mass Communicat Journalism 8: 373. doi: 10.4172/21657912.1000373

Copyright: ( 2018 Tareke G, et al. This is an open-access article distributed under the terms of the Creative Commons Attribution License, which permits unrestricted use, distribution, and reproduction in any medium, provided the original author and source are credited. 
addition information was gathered from principals and vice principals of the schools, supervisors, and zone and woreda education officers. The study was conducted in south Wollo Zone. A reason selecting the zone was that the researcher had some familiarity with teachers, supervisors and school principals in the area which provided ease of access to administer and collect the data from respondents.

\section{Sampling techniques}

Purposive and stratified random sampling techniques were employed to select the sample woredas, schools, and respondents. South Wollo zone has 20 woredas and 1 town administration (Dessie). The rest 20 woredas were classified as accessible and remote woredas. Considering the serious difficulty of reaching remote wordas, particularly the transportation problem and the limited budget available the researcher selected the sample only from accessible woredas. Accordingly one town administration (Dessie) and three woredas namely Alboko, Kutaber, Tehuledere were included in the study (Table 1).

Regarding supervisors, principals and vice principals of the schools, all supervisors, principals and vice principals in the sample primary schools included in the study.

Instruments of data collection: The data of the study were collected at different levels (zone, Wereda, and school) using different data collection instruments such as questionnaires, teacher self-efficacy scale, interview, observation and document analysis.

Questionnaires: Questionnaires were administered to teachers, principals, vice principal and supervisors. The questionnaires included both open and close ended questions. The close ended questions were prepared in the form of Likert scale type.

The questionnaires dealt with the practice CPD in the school, the effects of CPD on teachers' knowledge and skills (such as the practice of active learning methods, continuous assessment, classroom management skill and planning) and the perception of teachers regarding the benefits of $\mathrm{CPD}$.

Self-efficacy scale: Items pertaining to teacher sense of efficacy were adapted in part from Tschannen-Moran and Woolfok Hoy [1] and in part developed by the researcher. The teacher self-efficacy scales consisted of 30 items clustered around three dimensions such as efficacy for instructional strategies (10 items), efficacy for classroom management (10 items) and efficacy for student engagement (10 items). The 9-point continuum of the Tschannen-Moran and Woolfok Hoy [1] TSES were revised to have a 4-point Likert scale ranging from "very little" (1 point) to "a lot" (4 points). Interview

Semi- structure interview was conducted with zone and woreda education officers to obtain information regarding the implementation of CPD.

Document analysis: Portfolios kept by the teachers as a part of their CPD program were analyzed by preparing check list. The purpose of the document analysis was to find out if the portfolio is used by teachers as tool for professional development.

Observation: Observations of class sessions were made to obtain further information about teachers' implement CPD program by using observation checklist. The checklist consisted of topics taken from CPD content. In each school one class session observation was conducted.

Data analysis: Both qualitative and quantitative data analysis techniques were used to analyze the data. Information gathered through closed ended questionnaires was analyzed using frequency count, percentage, and analysis of variance. Data gathered through open ended questionnaire, interview and observation were analyzed qualitatively.

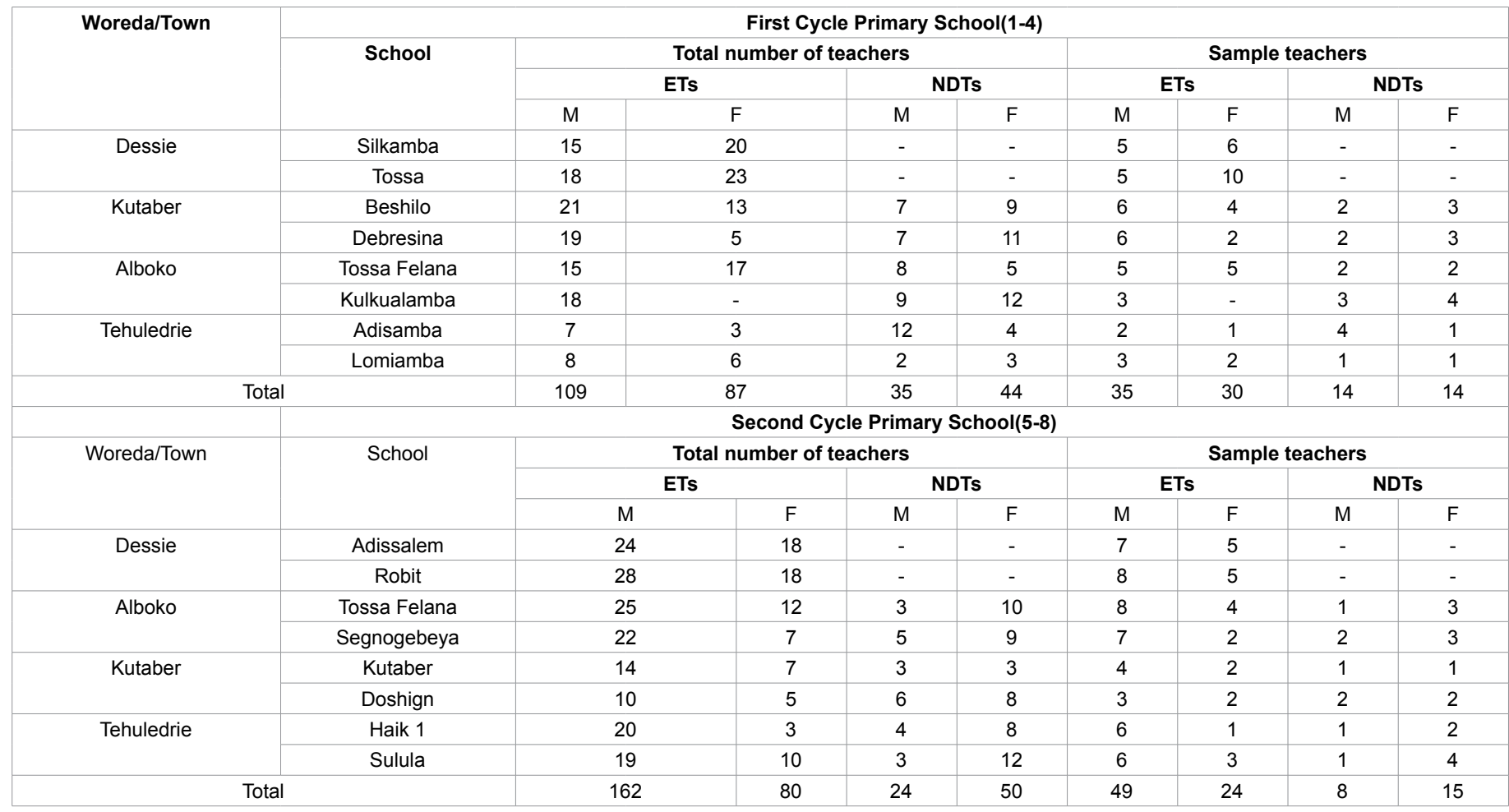

Table 1: The summary of research participants is presented in the table below. 
The research questions, data gathering tools and the data analysis techniques are shown as follow (2).

\section{Results}

\section{CPD training opportunity}

Teachers, principals and supervisors who had participated in the study were asked to evaluate the CPD training opportunities in their school. The result is summarized in table below (Table 2).

Table 3 depicts that $55.3 \%$ of first cycle primary school teachers consider the opportunities of CPD training is adequate while $59.3 \%$ of second cycle primary school teachers confirmed that CPD training opportunities were inadequate. On the other hand, the majority of supervisors and school principals (57.1\%) reported that the availability of good CPD training opportunities in their school. Thus it is possible to say that the CPD training opportunity in the school is inadequate as it stated by a considerable number of teachers. From this, it is possible to conclude that teachers believe there is no adequate CPD training opportunity to update their profession.

\section{Extent of teacher's participation in cpd learning activities}

Teachers, principals and supervisors were asked to rate the extent of teachers' participation in CPD learning activities. The responses obtained are presented as follow

Table 4 shows that the mean value for activities such as peer observation, team teaching, join lesson planning group discussion and portfolio were found are over the point of the rating scale (2.50). This indicates that most teachers often participated in group discussion, peer observation, joint lesson planning, maintaining portfolio and team teaching during CPD training. On the other hand, it was found out that teacher in the school under study participated in CPD activities some times and even not at all in some of the activities such as private reading, self-reflection, personal need assessment, workshop, experience sharing and demonstration of best practice. Similarly the interview conducted with woreda and zone education officer disclosed that teachers participation in the activities prescribed in CPD guideline is very low. One of the informants among the Woreda education officer said.

Table 5 as indicated in table above the mean values for most items are falling in high emphasis category. There it can be generalized that CPD training gives high emphasis for active learning methods, assessment techniques, action research, classroom management, lesson planning and pedagogical knowledge and skills. On the other hand the majority of the respondents confirmed that the CPD training of teachers gives no emphasis for subject matter knowledge and skills. However, research evidence confirmed that effective CPD program should give emphasis for teachers' subject matter knowledge [12-15] (Table 6).

The above table indicates that the majority of first cycle and second cycle primary school teachers, supervisors and principals replied positively on the value of CPD program to help teachers make educational discussion about their profession, to work in collaboration and to conduct collaborative action research, develop mutual trust, to involve in peer observation and evaluation and sharing of important educational resources like books and teaching aids. Thus one can say that most of the activities designed for CPD course encourage teachers to make educational discussion about their profession, to work in collaboration and make collegial relationship; to involve in peer observation and evaluation. Supporting this research evidence shows that teachers' professional development activities are most effective when it is carried out collaboratively in the atmosphere of mutual support and encouragement. The most important way to improve the skill of teaching is to learn from each other and share good practice with colleagues [16]. Teachers feel a sense of accomplishment in their development when they generate high level of collegiality, communication and trust among themselves [17]. Day (1999) also identified collegiality and collaboration as the top characteristics of effective and successful teacher professional development [18]. Harwell [13] also argued that learning is occurred in the context of sharing and communicating with other learners (Table 7).

\begin{tabular}{|c|c|c|c|}
\hline S no & Research questions & Data Gathering tools & Analysis Techniques \\
\hline 1 & $\begin{array}{c}\text { To what extent do schools implement CPD } \\
\text { program to develop teachers' profession? }\end{array}$ & $\begin{array}{c}\text { Questionnaire } \\
\text { Document analysis } \\
\text { Interview }\end{array}$ & $\begin{array}{c}\text { Frequency count; percentage } \\
\text { Questionnaire } \\
\text { Observation }\end{array}$ \\
\hline 2 & $\begin{array}{c}\text { To what extent do teachers implement CPD in } \\
\text { their classroom to improve students' learning } \\
\text { outcomes? }\end{array}$ & $\begin{array}{c}\text { Self-efficacy scale } \\
\text { of facets of CPD such as collaboration; maintaining portfolio }\end{array}$ \\
\hline 3 & $\begin{array}{c}\text { Are there statistically significant differences among } \\
\text { experienced and newly deployed; first and second } \\
\text { cycle primary school teachers) in terms of their } \\
\text { self-efficacy to implement CPD? }\end{array}$ & $\begin{array}{c}\text { Data will be analyzed by identifying themes on the practice of } \\
\text { facets of CPD such as active learning methods; continuous } \\
\text { assessment }\end{array}$ \\
\hline 4 & $\begin{array}{r}\text { Are there statistically significant differences among } \\
\text { experienced and newly deployed; first and second } \\
\text { cycle primary school teachers in their perception } \\
\text { about CPD? }\end{array}$ & Scale in questionnaire & Three Wanalysis of Variance/ANOVA/ \\
\hline
\end{tabular}

Table 2: Research questions and their data gathering tools and analysis techniques.

\begin{tabular}{|c|c|c|c|c|c|c|c|c|c|c|}
\hline \multirow[t]{3}{*}{ Item } & \multirow[t]{3}{*}{ Respondents } & \multicolumn{9}{|c|}{ Rating Scale } \\
\hline & & \multicolumn{2}{|c|}{ V. good } & \multicolumn{2}{|c|}{ Good } & \multicolumn{2}{|c|}{ Adequate } & \multicolumn{2}{|c|}{ Inadequate } & \multirow[t]{2}{*}{ M } \\
\hline & & No & $\%$ & no & $\%$ & no & $\%$ & no & $\%$ & \\
\hline \multirow{3}{*}{$\begin{array}{l}\text { How do you judge } \\
\text { the CPD training } \\
\text { opportunities in your } \\
\text { school? }\end{array}$} & Fcpt. & 3 & 3.5 & 15 & 17.6 & 47 & 55.3 & 20 & 23.5 & 2.11 \\
\hline & Scpt. & 2 & 2.2 & 0 & 0 & 36 & 38.7 & 55 & 59.3 & 1.45 \\
\hline & Ps. & 15 & 42.9 & 20 & 57.1 & 0 & 0 & 0 & 0 & 3.57 \\
\hline
\end{tabular}

Note: Fcpt: First cycle primary school teachers; Scpt: Second cycle primary school teachers; Ps: Principals and Supervisors.

Table 3: Teachers'; Principals' and Supervisors' Judgment of the Adequacy of CPD Training. 
Citation: Tareke G, Tefera B (2018) The Status and Psychological Challenges of Implementation of Continuous Professional Development/CPD/ in Primary Schools of South Wollo Zone. J Mass Communicat Journalism 8: 373. doi: 10.4172/2165-7912.1000373

Page 4 of 9

\begin{tabular}{|c|c|c|c|c|c|c|c|c|c|c|}
\hline \multirow[t]{3}{*}{ Activities } & \multirow[t]{3}{*}{ Respondents } & \multicolumn{9}{|c|}{ Rating Scale } \\
\hline & & \multicolumn{2}{|c|}{ Always } & \multicolumn{2}{|c|}{ Often } & \multicolumn{2}{|c|}{ S/times } & \multicolumn{2}{|c|}{ Not at all } & \multirow[t]{2}{*}{ M } \\
\hline & & No & $\%$ & no & $\%$ & no & $\%$ & no & & \\
\hline \multirow[t]{3}{*}{ Experience sharing } & Fcpt. & 3 & 3.5 & 15 & 17.6 & 40 & 47.1 & 27 & 31.8 & 1.93 \\
\hline & Scpt. & 0 & 0 & 11 & 11.8 & 55 & 59.2 & 27 & 29.0 & 1.82 \\
\hline & Ps. & 2 & 5.7 & 16 & 45.7 & 10 & 28.6 & 7 & 20.0 & 2.37 \\
\hline \multirow[t]{3}{*}{ Private reading/study } & Fcpt. & 11 & 12.9 & 18 & 21.2 & 33 & 38.8 & 18 & 21.2 & 2.20 \\
\hline & Scpt. & 0 & 0 & 23 & 24.7 & 63 & 67.9 & 7 & 7.5 & 2.17 \\
\hline & Ps. & 3 & 8.6 & 12 & 34.3 & 17 & 48.6 & 3 & 8.6 & 2.43 \\
\hline \multirow[t]{3}{*}{ Action research } & Fcpt. & 0 & 0 & 42 & 49.4 & 31 & 36.5 & 2 & 2.4 & 2.09 \\
\hline & Scpt. & 0 & 0 & 20 & 21.5 & 48 & 51.6 & 25 & 26.9 & 1.94 \\
\hline & Ps. & 0 & 0 & 8 & 17.1 & 21 & 60.0 & 8 & 22.9 & 1.82 \\
\hline \multirow[t]{3}{*}{ Self-reflection } & Fcpt & 7 & 8.2 & 11 & 12.9 & 61 & 71.8 & 13 & 15.3 & 1.97 \\
\hline & Scpt. & 0 & 0 & 17 & 18.3 & 64 & 68.8 & 12 & 12.9 & 2.05 \\
\hline & Ps. & 0 & 0 & 9 & 25.7 & 24 & 68.6 & 2 & 5.7 & 2.20 \\
\hline \multirow[t]{3}{*}{ Peer observation } & Fcpt & 5 & 5.9 & 42 & 49.4 & 35 & 41.2 & 3 & 3.5 & 2.58 \\
\hline & Scpt & 8 & 8.6 & 39 & 41.9 & 40 & 43.06 & 6 & 6.5 & 2.53 \\
\hline & Ps. & 5 & 14.3 & 12 & 34.3 & 18 & 51.4 & 0 & 0 & 2.63 \\
\hline \multirow[t]{3}{*}{ Workshop/seminar/ } & Fcpt & 0 & 0 & 3 & 3.5 & 58 & 68.2 & 24 & 28.2 & 1.75 \\
\hline & Scpt. & 0 & 0 & 7 & 7.5 & 64 & 68.8 & 22 & 23.7 & 1.84 \\
\hline & Ps. & 8 & 22.9 & 20 & 57.1 & 7 & 20.0 & 0 & 0 & 2.03 \\
\hline \multirow[t]{3}{*}{ Team teaching } & Fcpt & 17 & 20.0 & 37 & 43.5 & 26 & 30.6 & 5 & 5.9 & 2.77 \\
\hline & Scpt. & 5 & 5.4 & 54 & 58.1 & 32 & 34.4 & 2 & 2.1 & 2.67 \\
\hline & Ps. & 3 & 8.6 & 17 & 48.6 & 13 & 37.1 & 2 & 5.7 & 2.60 \\
\hline \multirow[t]{3}{*}{ Joint lesson planning } & Fcpt & 22 & 25.9 & 35 & 41.2 & 26 & 30.6 & 2 & 2.4 & 2.91 \\
\hline & Scpt. & 7 & 7.5 & 53 & 57.0 & 33 & 35.5 & 0 & 0 & 2.72 \\
\hline & Ps. & 10 & 28.6 & 19 & 54.3 & 6 & 17.1 & 0 & 0 & 3.11 \\
\hline \multirow[t]{3}{*}{ Demonstration of best practice } & Fcpt & 0 & 0 & 14 & 16.5 & 51 & 60.0 & 20 & 23.5 & 1.92 \\
\hline & Scpt. & 0 & 0 & 16 & 17.2 & 44 & 47.3 & 33 & 35.5 & 1.83 \\
\hline & Ps. & 0 & 0 & 8 & 22.9 & 16 & 30.2 & 11 & 31.4 & 1.92 \\
\hline \multirow[t]{3}{*}{ Discussion } & Fcpt & 35 & 41.2 & 25 & 29.4 & 12 & 14.1 & 13 & 15.3 & 2.96 \\
\hline & Scpt. & 24 & 25.8 & 41 & 44.1 & 26 & 28.0 & 2 & 2.2 & 2.94 \\
\hline & Ps. & 10 & 28.8 & 19 & 35.8 & 6 & 17.1 & 0 & 0 & 3.12 \\
\hline \multirow[t]{3}{*}{ Personal need assessment } & Fcpt. & 0 & 0 & 14 & 16.5 & 56 & 65.9 & 15 & 17.6 & 2.60 \\
\hline & Scpt. & 24 & 25.8 & 34 & 36.6 & 23 & 24.7 & 12 & 12.9 & 2.75 \\
\hline & Ps. & 6 & 17.1 & 7 & 20.0 & 18 & 51.4 & 4 & 11.4 & 2.43 \\
\hline \multirow[t]{3}{*}{ Portfolio } & Fcpt. & 49 & 57.5 & 30 & 35.5 & 5 & 5.3 & 1 & 1.2 & 3.49 \\
\hline & Scpt. & 32 & 34.4 & 42 & 45.7 & 17 & 18.3 & 2 & 2.2 & 3.12 \\
\hline & Ps. & 19 & 25.4 & 14 & 40.0 & 2 & 5.7 & 0 & 0 & 3.49 \\
\hline
\end{tabular}

Note: Fcpt: First cycle primary school teachers; Scpt-second cycle primary school teachers; Ps: Principals and Supervisors.

Table 4: Kinds of Learning Activity and Teachers' participation in them.

\begin{tabular}{|c|c|c|c|c|c|c|c|c|c|c|}
\hline \multirow[t]{3}{*}{ Item } & \multirow[t]{3}{*}{ Respondents } & \multicolumn{9}{|c|}{ Rating Scale } \\
\hline & & \multicolumn{2}{|c|}{ V. high emphasis } & \multicolumn{2}{|c|}{ High emphasis } & \multicolumn{2}{|c|}{ Low emphasis } & \multicolumn{2}{|c|}{ No emphasis } & \multirow[t]{2}{*}{$\mathbf{M}$} \\
\hline & & No & $\%$ & no & $\%$ & no & $\%$ & no & $\%$ & \\
\hline \multirow[t]{2}{*}{ Pedagogical knowledge and skill } & Fcpt. & 49 & 57.6 & 35 & 41.2 & 1 & 1.2 & 0 & 0 & 3.56 \\
\hline & Scpt. & 19 & 20.4 & 50 & 53.8 & 22 & 23.7 & 2 & 2.2 & 2.92 \\
\hline \multirow[t]{2}{*}{ Active teaching and learning methods } & Fcpt. & 13 & 15.3 & 63 & 74.1 & 9 & 10.8 & 0 & 0 & 3.04 \\
\hline & Scpt. & 26 & 28.0 & 52 & 55.9 & 13 & 14.0 & 2 & 2.2 & 3.09 \\
\hline \multirow[t]{2}{*}{ Assessment techniques } & Fcpt. & 32 & 37.6 & 35 & 41.2 & 11 & 12.9 & 7 & 8.2 & 3.08 \\
\hline & Scpt. & 25 & 26.9 & 45 & 48.4 & 21 & 22.6 & 2 & 2.2 & 3.00 \\
\hline \multirow[t]{2}{*}{ Action research } & Fcpt. & 32 & 37.6 & 47 & 55.3 & 5 & 5.9 & 1 & 1.2 & 3.29 \\
\hline & Scpt. & 27 & 29.0 & 43 & 46.2 & 18 & 19.4 & 5 & 5.4 & 2.99 \\
\hline \multirow[t]{2}{*}{ Class room managements } & Fcpt. & 28 & 32.9 & 34 & 40.0 & 19 & 22.4 & 4 & 4.7 & 3.01 \\
\hline & Scpt. & 24 & 25.8 & 46 & 49.5 & 19 & 20.4 & 4 & 4.3 & 2.97 \\
\hline \multirow[t]{2}{*}{ Lesson planning } & Fcpt. & 32 & 37.6 & 47 & 55.3 & 5 & 5.9 & 1 & 1.2 & 3.29 \\
\hline & Scpt. & 27 & 29.0 & 43 & 46.2 & 18 & 19.4 & 5 & 5.4 & 2.99 \\
\hline \multirow[t]{2}{*}{ Subject matter knowledge and skills } & Fcpt. & 16 & 18.8 & 18 & 18.8 & 25 & 29.4 & 44 & 51.8 & 1.67 \\
\hline & Scpt. & 0 & 0 & 20 & 21.5 & 34 & 36.6 & 39 & 41.9 & 1.79 \\
\hline
\end{tabular}

Note. Fcpt: First cycle primary school teachers; Scpt-second cycle primary school teachers; Ps: Principals and Supervisors.

Table 5: The Focus of CPD Training: Teachers Response Regarding the Focus of their CPD Training. 
Citation: Tareke G, Tefera B (2018) The Status and Psychological Challenges of Implementation of Continuous Professional Development/CPD/ in Primary Schools of South Wollo Zone. J Mass Communicat Journalism 8: 373. doi: 10.4172/2165-7912.1000373

Page 5 of 9

\begin{tabular}{|c|c|c|c|c|c|c|c|c|c|c|}
\hline \multirow[t]{2}{*}{ Tem } & \multirow[t]{3}{*}{ Respondents } & \multicolumn{8}{|c|}{ Options } & \multirow[t]{3}{*}{ M } \\
\hline & & \multicolumn{2}{|c|}{ S/agree } & \multicolumn{2}{|c|}{ Agree } & \multicolumn{2}{|c|}{ Disagree } & \multicolumn{2}{|c|}{ S/disagree } & \\
\hline Teachers: & & no & $\%$ & no & $\%$ & no & $\%$ & no & $\%$ & \\
\hline \multirow[t]{3}{*}{ Make profession discussion. } & Fcpt. & 19 & 22.4 & 36 & 42.4 & 28 & 32.9 & 2 & 2.4 & 2.85 \\
\hline & Scpt. & 17 & 18.3 & 43 & 46.2 & 27 & 29.0 & 6 & 6.5 & 2.76 \\
\hline & Ps. & 3 & 8.6 & 16 & 45.7 & 15 & 42.9 & 1 & 2.9 & 2.60 \\
\hline \multirow{3}{*}{$\begin{array}{l}\text { Work in collaboration and } \\
\text { coordination. }\end{array}$} & Fcpt. & 28 & 32.9 & 30 & 35.3 & 22 & 25.9 & 5 & 5.9 & 2.95 \\
\hline & Scpt. & 28 & 30.1 & 30 & 32.3 & 23 & 24.7 & 12 & 12.9 & 2.79 \\
\hline & Ps. & 2 & 5.7 & 20 & 57.1 & 12 & 34.3 & 1 & 2.9 & 2.66 \\
\hline \multirow{3}{*}{$\begin{array}{c}\text { Share important educational } \\
\text { resources such as book } \\
\text { teaching aids etc. }\end{array}$} & Fcpt. & 18 & 21.2 & 25 & 29.4 & 42 & 49.4 & 0 & 0 & 2.71 \\
\hline & Scpt. & 15 & 16.1 & 47 & 50.5 & 27 & 29.0 & 4 & 4.3 & 2.78 \\
\hline & Ps. & 4 & 11.4 & 14 & 40.0 & 17 & 48.6 & 0 & 0 & 2.63 \\
\hline \multirow{3}{*}{$\begin{array}{l}\text { Conduct collaborative action } \\
\text { research. }\end{array}$} & Fcpt. & 9 & 10.6 & 45 & 52.9 & 28 & 32.9 & 3 & 3.5 & 2.71 \\
\hline & Scpt. & 13 & 14.0 & 31 & 33.3 & 39 & 41.9 & 10 & 10.8 & 2.50 \\
\hline & Ps. & 4 & 11.4 & 18 & 51.4 & 9 & 25.7 & 4 & 11.4 & 2.62 \\
\hline \multirow[t]{3}{*}{ Developing mutual trust. } & Fcpt. & 15 & 17.6 & 54 & 63.5 & 15 & 17.6 & 1 & 1.2 & 2.97 \\
\hline & Scpt. & 7 & 7.5 & 43 & 46.2 & 36 & 38.7 & 7 & 7.5 & 2.54 \\
\hline & Ps. & 13 & 37.1 & 16 & 45.7 & 6 & 17.1 & 0 & 0 & 3.20 \\
\hline \multirow{3}{*}{$\begin{array}{l}\text { Involve in peer observation } \\
\text { and evaluation }\end{array}$} & Fcpt. & 29 & 34.1 & 44 & 51.8 & 6 & 7.1 & 6 & 7.1 & 3.13 \\
\hline & Scpt. & 29 & 30.2 & 43 & 44.8 & 18 & 19.4 & 3 & 3.2 & 3.05 \\
\hline & Ps. & 8 & 22.9 & 25 & 71.4 & 2 & 5.7 & 0 & 0 & 3.17 \\
\hline \multirow{3}{*}{$\begin{array}{l}\text { Show responsibility to } \\
\text { support new teachers }\end{array}$} & Fcpt. & 10 & 11.8 & 39 & 45.9 & 18 & 21.2 & 18 & 21.2 & 2.48 \\
\hline & Scpt. & 12 & 12.9 & 37 & 39.8 & 34 & 36.6 & 10 & 10.8 & 2.55 \\
\hline & Ps. & 2 & 5.7 & 16 & 45.7 & 17 & 48.6 & 0 & 0 & 2.57 \\
\hline
\end{tabular}

Note. Fcpt: First cycle primary school teachers; Scpt-second cycle primary school teachers; Ps: Principals and Supervisors.

Table 6: Impact of CPD to strengthen Collaboration: Teachers'; Principals' and Supervisors' Response Regarding the Impact of CPD to Strengthen Collaboration.

\begin{tabular}{|c|c|c|c|c|c|c|c|c|c|c|}
\hline \multirow[t]{3}{*}{ Item } & \multirow[t]{3}{*}{ Respondents } & \multicolumn{8}{|c|}{ Rating Scale } & \multirow{3}{*}{ M } \\
\hline & & \multicolumn{2}{|c|}{ S/agree } & \multicolumn{2}{|c|}{ Agree } & \multicolumn{2}{|c|}{ Disagree } & \multicolumn{2}{|c|}{ S/disagree } & \\
\hline & & no & $\%$ & no & $\%$ & no & $\%$ & no & & \\
\hline \multirow{3}{*}{$\begin{array}{l}\text { Teachers maintain and } \\
\text { prepare portfolio for } \\
\text { their CPD training }\end{array}$} & Fcpt. & 40 & 47.1 & 37 & 43.5 & 7 & 8.2 & 1 & 1.2 & 3.36 \\
\hline & Scpt. & 32 & 34.4 & 42 & 45.2 & 19 & 20.4 & 0 & 0 & 3.14 \\
\hline & Ps. & 12 & 34.3 & 19 & 54.3 & 4 & 11.4 & 0 & 0 & 3.23 \\
\hline \multirow{3}{*}{$\begin{array}{l}\text { Teachers invite others } \\
\text { teachers to observe } \\
\text { their CPD portfolio and } \\
\text { discuss the issue on it }\end{array}$} & Fcpt. & 12 & 14.1 & 55 & 64.7 & 18 & 21.2 & 0 & 0 & 2.92 \\
\hline & Scpt. & 8 & 8.6 & 37 & 39.8 & 38 & 40.9 & 10 & 10.8 & 2.46 \\
\hline & Ps. & 7 & 20.0 & 20 & 57.1 & 6 & 17.1 & 2 & 5.7 & 2.91 \\
\hline
\end{tabular}

Note. Fcpt: First cycle primary school teachers; Scpt-second cycle primary school teachers; Ps: Principals and Supervisors.

Table 7: Use of Portfolio: Teacher Response Regarding the Use of Portfolio during CPD Training

As can be seen in Table 8 teachers, school principals and supervisors strongly agree that portfolio is prepared as part of CPD training. $57.1 \%$ of School principal and supervisors and $64.7 \%$ first cycle primary school teachers replied that teacher invite other teachers to observe their portfolio and make discussion on it.

These finding correspond with the results of the document analysis. The document analysis confirmed that in all visited schools every teacher has his/her own individual CPD portfolio with most frequent mentioned topics such as application of continuous assessment, active learning methods, action research attempted and making teaching aids. But the finding form the document analysis revealed that in a number of cases teachers share their portfolio. But they did it not for the sake of discussion rather to copy text from each other's.

Many of the woreda and zone education officers complained about the teachers' portfolio. One of the informants among the zone education officers said:

Teachers have no knowledge about why and how to write their portfolio. Many teachers are just filling in the format because they think it is compulsory. In addition feedback was not given by principals and supervisors. Teachers write their portfolio and principals and supervisors simply sign on it without writing feedback. /South wolle zone education office.

The finding of the document analysis also indicates that no feedback is clearly written by principal and supervisor. Teachers copy what other teachers write and attach it to their portfolio. Form this one can conclude that portfolio is not considered as a means of improving teachers' profession.

However, Bush reported the use of portfolios to support teachers' professional development as they provide an opportunity for teachers to reflect on their activities in and outside the classroom. Craft (2004) also argued that developing portfolio is a powerful learning activity as teachers reflect on their teaching practice in light of standards [19]. Besides presenting one's portfolio to a group or meeting can make portfolio a powerful venue for CPD.

\section{Contribution of cpd for teaching learning process in the classroom}

Teachers are expected to implement what is practiced in their 
Citation: Tareke G, Tefera B (2018) The Status and Psychological Challenges of Implementation of Continuous Professional Development/CPD/ in Primary Schools of South Wollo Zone. J Mass Communicat Journalism 8: 373. doi: 10.4172/2165-7912.1000373

Page 6 of 9

\begin{tabular}{|c|c|c|c|c|c|c|c|c|c|c|}
\hline \multirow[t]{3}{*}{ Item } & \multirow[t]{3}{*}{ Respondents } & \multicolumn{9}{|c|}{ Rating scale } \\
\hline & & \multicolumn{2}{|c|}{ G. extent } & \multicolumn{2}{|c|}{ S. extent } & \multicolumn{2}{|c|}{ L. extent } & \multicolumn{2}{|c|}{ N. at all } & \multirow[t]{2}{*}{$M$} \\
\hline & & no & $\%$ & no & $\%$ & no & $\%$ & no & $\%$ & \\
\hline \multirow{3}{*}{$\begin{array}{l}\text { Practicing a wide varieties } \\
\text { of active teaching and } \\
\text { learning methods }\end{array}$} & Fcpt. & 10 & 11.8 & 64 & 75.3 & 11 & 12.9 & 0 & 0 & 2.98 \\
\hline & Scpt. & 14 & 15.1 & 53 & 57.0 & 21 & 22.6 & 5 & 5.4 & 2.81 \\
\hline & Ps. & 7 & 20.0 & 28 & 80.0 & 0 & 0 & 0 & 0 & 3.20 \\
\hline \multirow{3}{*}{$\begin{array}{l}\text { Implementing various } \\
\text { techniques of continuous } \\
\text { assessment }\end{array}$} & Fcpt. & 15 & 17.6 & 70 & 82.4 & 0 & 0 & 0 & 0 & 3.18 \\
\hline & Scpt. & 23 & 24.7 & 47 & 50.5 & 20 & 21.5 & 3 & 3.2 & 2.96 \\
\hline & Ps. & 11 & 31.4 & 18 & 51.4 & 5 & 14.3 & 1 & 2.9 & 3.11 \\
\hline \multirow{3}{*}{$\begin{array}{l}\text { Promoting the culture } \\
\text { of group work and } \\
\text { collaboration }\end{array}$} & Fcpt. & 20 & 23.5 & 53 & 62.4 & 10 & 11.8 & 2 & 2.4 & 3.07 \\
\hline & Scpt. & 15 & 16.1 & 40 & 43.0 & 35 & 37.6 & 3 & 3.2 & 2.72 \\
\hline & Ps. & 7 & 20.0 & 25 & 71.4 & 3 & 8.6 & 0 & 0 & 3.12 \\
\hline \multirow{3}{*}{$\begin{array}{l}\text { Better management of } \\
\text { classroom }\end{array}$} & Fcpt. & 20 & 23.5 & 58 & 68.2 & 7 & 8.2 & 0 & 0 & 3.15 \\
\hline & Scpt. & 21 & 22.6 & 35 & 37.6 & 32 & 34.4 & 5 & 5.2 & 2.77 \\
\hline & Ps. & 9 & 25.7 & 21 & 60.0 & 5 & 14.3 & 0 & 0 & 3.14 \\
\hline \multirow{3}{*}{$\begin{array}{l}\text { Preparation of good lesson } \\
\text { planning }\end{array}$} & Fcpt. & 36 & 42.4 & 41 & 48.2 & 8 & 9.4 & 0 & 0 & 3.32 \\
\hline & Scpt. & 43 & 46.2 & 30 & 32.3 & 18 & 19.4 & 2 & 2.2 & 3.22 \\
\hline & Ps. & 15 & 42.9 & 15 & 42.9 & 4 & 11.4 & 1 & 2.9 & 3.25 \\
\hline \multirow{3}{*}{$\begin{array}{l}\text { Using locally produced and } \\
\text { appropriate teaching aids }\end{array}$} & Fcpt. & 29 & 34.1 & 40 & 57.6 & 6 & 7.1 & 1 & 1.2 & 3.24 \\
\hline & Scpt. & 38 & 40.9 & 34 & 36.6 & 19 & 20.4 & 2 & 2.2 & 3.16 \\
\hline & Ps. & 6 & 17.1 & 11 & 31.4 & 13 & 37.1 & 5 & 14.3 & 2.51 \\
\hline \multirow{3}{*}{$\begin{array}{l}\text { Conducting action } \\
\text { research to solve problems } \\
\text { related to the teaching } \\
\text { learning process. }\end{array}$} & Fcpt. & 28 & 32.9 & 32 & 37.6 & 20 & 23.5 & 5 & 5.9 & 2.97 \\
\hline & Scpt. & 20 & 21.5 & 44 & 47.3 & 29 & 31.2 & 0 & 0 & 2.90 \\
\hline & Ps. & 10 & 28.6 & 13 & 37.1 & 9 & 25.7 & 3 & 8.6 & 2.86 \\
\hline
\end{tabular}

Note. Fcpt: First cycle primary school teachers; Scpt-second cycle primary school teachers; Ps: Principals and Supervisors.

Table 8: Effects of CPD on Classroom Tasks.

\begin{tabular}{|c|c|c|c|c|c|c|c|c|c|c|}
\hline \multirow[t]{3}{*}{ Item } & \multirow[t]{3}{*}{ Respondents } & \multicolumn{8}{|c|}{ Options } & \multirow[t]{3}{*}{ M } \\
\hline & & \multicolumn{2}{|c|}{ S. agree } & \multicolumn{2}{|c|}{ Agree } & \multicolumn{2}{|c|}{ Disagree } & \multicolumn{2}{|c|}{ S. disagree } & \\
\hline & & No & $\%$ & no & $\%$ & no & $\%$ & no & & \\
\hline \multirow{2}{*}{$\begin{array}{l}\text { There are adequate facilities budget } \\
\text { and training materials for CPD }\end{array}$} & Fcpt. & 7 & 8.2 & 24 & 29.2 & 47 & 55.5 & 7 & 8.2 & 2.36 \\
\hline & Scpt. & 12 & 12.9 & 31 & 33.3 & 39 & 41.9 & 11 & 11.8 & 2.45 \\
\hline \multirow{2}{*}{$\begin{array}{l}\text { There is responsible body to } \\
\text { coordinate CPD activities. }\end{array}$} & Fcpt. & 6 & 7.1 & 26 & 30.6 & 52 & 61.2 & 1 & 1.2 & 2.43 \\
\hline & Scpt. & 9 & 9.7 & 34 & 36.6 & 44 & 47.3 & 6 & 6.5 & 2.50 \\
\hline \multirow{2}{*}{$\begin{array}{l}\text { There is established system for } \\
\text { experience sharing in the school }\end{array}$} & Fcpt. & 3 & 3.5 & 14 & 16.5 & 69 & 80.0 & 0 & 0 & 2.23 \\
\hline & Scpt. & 30 & 32.3 & 22 & 23.7 & 39 & 41.9 & 2 & 2.2 & 2.65 \\
\hline \multirow{2}{*}{$\begin{array}{l}\text { The school principal evaluate and give } \\
\text { constructive feedback after observing } \\
\text { CPD portfolio }\end{array}$} & Fcpt. & 2 & 2.4 & 32 & 37.6 & 36 & 42.4 & 15 & 17.9 & 2.25 \\
\hline & Scpt. & 5 & 5.4 & 36 & 38.7 & 39 & 41.9 & 13 & 14.0 & 2.38 \\
\hline \multirow{2}{*}{$\begin{array}{l}\text { The school principal encourage inter } \\
\text { school experience sharing }\end{array}$} & Fcpt. & 20 & 23.5 & 37 & 43.5 & 22 & 25.9 & 6 & 7.1 & 2.83 \\
\hline & Scpt. & 30 & 32.3 & 46 & 49.5 & 13 & 14.0 & 4 & 4.2 & 3.09 \\
\hline \multirow{2}{*}{$\begin{array}{l}\text { The school principal facilitates } \\
\text { teachers to make peer evaluation }\end{array}$} & Fcpt. & 18 & 21.5 & 27 & 31.8 & 36 & 42.4 & 4 & 4.7 & 2.80 \\
\hline & Scpt. & 9 & 9.4 & 32 & 34.4 & 41 & 44.1 & 11 & 11.6 & 2.41 \\
\hline \multirow{2}{*}{$\begin{array}{l}\text { The school principal conducts } \\
\text { observation during discussion and } \\
\text { peer evaluation }\end{array}$} & Fcpt. & 9 & 10.6 & 35 & 41.2 & 28 & 32.9 & 13 & 15.3 & 2.47 \\
\hline & Scpt. & 7 & 7.5 & 30 & 32.3 & 37 & 39.8 & 19 & 20.4 & 2.27 \\
\hline
\end{tabular}

Note. Fcpt: First cycle primary school teachers; Scpt-second cycle primary school teachers.

Table 9: Teachers evaluation of the role of leadership support to implement CPD.

$\mathrm{CPD}$ in to their classroom to enhance student learning. During data gathering process an attempt was made to obtained data regarding the effect of CPD on teachers' classroom instruction (Table 9).

As indicated in Table 10 the rating mean for each item revealed that the CPD program helped teachers to some extent in implementing continuous assessment, practicing active learning methods, classroom management, preparing of lesson plan and promoting the culture of group work and collaboration among students. Thus it is possible to say that CPD activities have contributed positively to teachers day to day activities even if not to the greater extent.

In addition to this, interview were made with the woreda and zone education expert to know the types of improvement teachers made in their day to day work after the implementation of CPD program. One of the informants reported that:

Actually some improvements were observed on activities such as implementing active learning methods, continuous assessment, classroom managements, developing sense of group work, problem solving skills, lesson plan preparation./Kutaber woreda education expert.

However other informants gave contrary ideas by saying: Teachers in our school think that CPD is something that has relationship with politics. They didn't consider CPD as relevant to classroom practice. For them, it is an imposition by the government. 
As result they showed no significant improvement concerning their classroom practice. /Dessie ketema education officer.

On the other hand, teachers frequently reported that CPD training helped them to apply different active learning methods and develop their knowledge and skill on how to do continuous assessment, prepare daily and annual lesson plan, and to conduct action research to solve classroom related problems.

However, class session observation also revealed that teachers used limited type of active learning methods such as group work, question and answer and lecture method. Among these group work is the most frequently used teaching method. Table and student seats are arranged in group and student seem to have experience in group work. The same pattern is observed in other teaching skills such as use of teaching aids, assessment practice and lesson planning. For example teachers rely only on tests and oral question, while ignoring other continuous assessment techniques such as quizzes, class work, homework, classroom observation etc. The classroom has no display. Teachers rarely used teaching aids during their lesson. Thus, there is a discrepancy between what teachers, principals and supervisors said and lesson observation about the practice of CPD in the classroom to enhance student outcome. In the lesson observation active learning methods, assessment techniques, and teaching aids are seldom seen whereas teachers and other respondents say that teachers implement them in their classroom most of the time. The above rival ideas clearly indicate that the CPD program did not bring the intended results in classroom and its implementation also lucks practicality.

\section{The role of school principals during cpd implementation}

Teaches were asked to evaluate the role of school leadership in planning, facilitating, coordinating supporting and evaluating the practice of CPD. The response obtained is summarized as follow.

The first items in Table 9 examined whether the school leaders played the role by providing the required facilities for CPD activities. The rating mean of first cycle primary school teachers (2.36) and second cycle primary school teachers (2.45) show that there were no appropriate facilities and budget to undertake CPD program and the supports given was insufficient. Based on this idea, one can say that the support given by the school principal was not up to the extent of expectation.
For other items that assessed whether the school leaders, evaluate teacher portfolio and give timely feedback and comments and whether they were in a position to create responsible body to coordinate CPD activities. As the rating means these items show that the respondents reacted negatively to the issues. Therefore, is it is possible to conclude that that the school principals were less effective in conducting follow up activities ,provide constructive feedback and take remedial action on time and to play their roles meaningfully to create responsible body to coordinate the CPD activities (Table 11).

Teachers' perceptions regarding the relevance CPD program: The above table shows that there is statistically significant difference between first and second cycle primary school teachers in their perception of CPD, $\mathrm{F}(1,178)=42.492, \mathrm{P}<0.05$. Form this; we can infer that first cycle primary school teachers have positive attitude towards CPD training than second cycle primary school teachers and already know the advantage of CPD training. In addition there is statistically significant difference between experienced and newly qualified teachers in their perception of $\mathrm{CPD}, \mathrm{F}(1,178)=11.370, \mathrm{P}<0.05$. This shows that newly qualified teachers highly participated in professional development activities.

Supporting this, Smith and Hofer (2002) identified the following individual characteristics as influencing how much and in what ways, teachers changed after participating in professional development: those teachers with fewer years of experience and teachers with low level of education changed more [20]. Bennet et al. found that teachers nearer to retirement are less likely to accept suggestion for change [21]. Newly qualified teachers have desire to collect as many experiences and ideas as possible and adapt and use new approaches most effectively in their teaching (Table 12).

Teachers' self-efficacy to practice cpd in the classroom: Teacher self-efficacy regarding instruction; Table 13 shows that there is statistically significant difference between first and second cycle primary school teachers in their self-efficacy regarding instruction, $\mathrm{F}(1,178)=28.753, \mathrm{P}<0.05$. Similarly there is statistically significant difference between male and female teachers' self-efficacy regarding instruction, $\mathrm{F}(1,178)=8.491, \mathrm{P}<0.05$. This leads to the conclusion

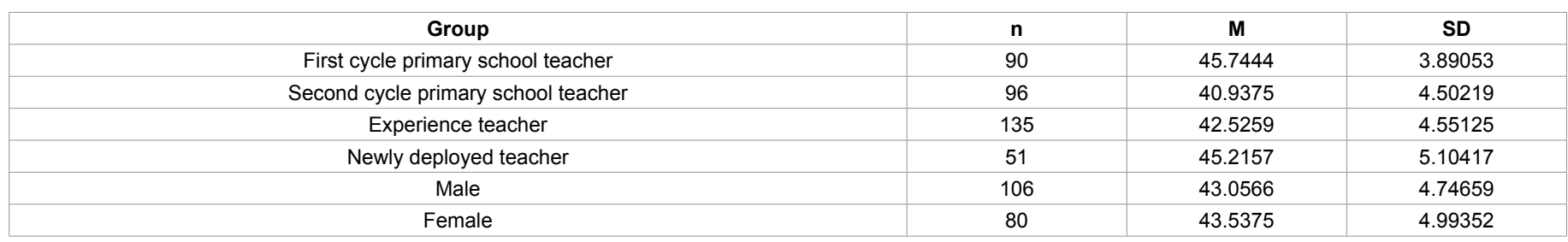

Table 10: Mean and Standard Deviation of Male; Female; Experienced; Newly Deployed; First and Second Cycle Primary School Teachers Perception of CPD.

\begin{tabular}{|c|c|c|c|c|}
\hline Source of variation & Sum of Squares & df & Mean Square & $\mathbf{F}$ \\
\hline School type & 732.767 & 1 & 732.767 & $42.492^{*}$ \\
\hline Experience & 196.079 & 1 & 196.079 & $11.370^{*}$ \\
\hline Gender & 1.919 & 1 & 1.919 & .111 \\
\hline School type $\times$ Experience & .003 & 1 & .003 & .000 \\
\hline School type $\times$ gender & 9.297 & 1 & 9.297 & .539 \\
\hline Experience $\times$ gender & 1.851 & 1 & 1.851 & .107 \\
\hline School type $\times$ Experience $\times$ gender & 6.961 & 1 & 6.961 & .404 \\
\hline Error & 3069.544 & 178 & 17.245 & \\
\hline Total & 352487.000 & 186 & & \\
\hline
\end{tabular}

${ }^{*} \mathrm{P}<0.05$

Table 11: Summary of ANOVA: The Significance of Mean Difference of Teachers' Perception of CPD with Reference to School type; Experience and Sex. 
Citation: Tareke G, Tefera B (2018) The Status and Psychological Challenges of Implementation of Continuous Professional Development/CPD/ in Primary Schools of South Wollo Zone. J Mass Communicat Journalism 8: 373. doi: 10.4172/2165-7912.1000373

Page 8 of 9

\begin{tabular}{|c|c|c|c|c|c|c|c|}
\hline \multirow[t]{3}{*}{ Group } & \multirow[t]{3}{*}{$\mathbf{n}$} & \multicolumn{6}{|c|}{ Dimensions of teacher self-efficacy } \\
\hline & & \multicolumn{2}{|c|}{ Efficacy regarding instruction } & \multicolumn{2}{|c|}{$\begin{array}{c}\text { Efficacy regarding classroom } \\
\text { management }\end{array}$} & \multicolumn{2}{|c|}{$\begin{array}{c}\text { Efficacy regarding student } \\
\text { engagement }\end{array}$} \\
\hline & & M & SD & $\mathrm{M}$ & SD & M & SD \\
\hline First cycle primary school teacher & 90 & 19.82 & 2.30 & 21.81 & 2.32 & 20.17 & 1.82 \\
\hline Second cycle primary school teacher & 96 & 19.93 & 2.35 & 20.95 & 1.74 & 20.37 & 1.81 \\
\hline Experience teacher & 135 & 20.02 & 2.51 & 21.54 & 2.20 & 20.47 & 1.93 \\
\hline Newly deployed teacher & 51 & 19.49 & 1.70 & 20.90 & 1.64 & 19.76 & 1.36 \\
\hline Male & 106 & 20.08 & 2.11 & 21.50 & 2.22 & 20.25 & 1.83 \\
\hline Female & 80 & 19.63 & 2.57 & 21.18 & 1.86 & 20.31 & 1.73 \\
\hline
\end{tabular}

Table 12: Mean and Standard Deviation of Male; Female; Experienced; Newly Deployed; First and Second Cycle Primary School Teacher self-efficacy.

\begin{tabular}{|c|c|c|c|c|}
\hline Source of variation & Sum of Squares & df & Mean Square & $\mathbf{F}$ \\
\hline School type & 190.618 & 1 & 190.618 & $28.753^{*}$ \\
\hline Experience & 0.187 & 1 & 0.187 & 0.028 \\
\hline Gender & 56.289 & 1 & 56.289 & $8.491^{*}$ \\
\hline School type $\times$ Experience & 1.044 & 1 & 1.044 & 0.157 \\
\hline School type $\times$ gender & 2.952 & 1 & 2.952 & 0.445 \\
\hline Experience $\times$ gender & 15.487 & 1 & 15.487 & 2.336 \\
\hline School type $\times$ Experience $\times$ gender & 15.292 & 1 & 15.292 & 2.307 \\
\hline Error & 1180.048 & 178 & 6.629 & \\
\hline Total & 84420.000 & 186 & & \\
\hline
\end{tabular}

${ }^{*} \mathrm{P}<0.05$

Table 13: Summary of ANOVA: The Significance of Mean Difference of Teachers' Self -efficacy Regarding Instruction.

\begin{tabular}{|c|c|c|c|c|}
\hline Source of variation & Sum of Squares & df & Mean Square & $\mathbf{F}$ \\
\hline School type & 11.147 & 1 & 11.147 & 2.909 \\
\hline Experience & 18.939 & 1 & 18.939 & $4.942^{*}$ \\
\hline Gender & 1.482 & 1 & 1.482 & 0.387 \\
\hline School type $\times$ Experience & 8.355 & 1 & 8.355 & 2.180 \\
\hline School type $\times$ gender & 24.052 & 1 & 24.052 & $6.277^{*}$ \\
\hline Experience $\times$ gender & 2.591 & 1 & 2.591 & 0.676 \\
\hline School type $\times$ Experience $\times$ gender & 2.591 & 1 & 2.591 & 0.676 \\
\hline Error & 682.091 & 178 & 3.832 & \\
\hline Total & 85706.000 & 186 & & \\
\hline
\end{tabular}

${ }^{*} \mathrm{P}<0.05$

Table 14: Summary of ANOVA: The significance of Mean Difference of Teachers' Self: efficacy Regarding Classroom Management.

that teachers from second cycle primary school had better classroom instruction self-efficacy.

Teacher self-efficacy regarding classroom management: Table 14 indicates there is statistically significant difference between newly deployed and experienced teachers in their self-efficacy regarding classroom management, $\mathrm{F}(1,178)=4.942, \mathrm{P}<0.05$. This shows that experienced teachers had better classroom management self-efficacy. In addition there is also significant interaction between school type and gender, $\mathrm{F}(1,178)=6.277, \mathrm{P}<0.05$.

Teacher self-efficacy regarding student engagement: Table 15 shows that there is statistically significant difference between newly deployed and experienced teachers in their self-efficacy regarding student engagement, $\mathrm{F}(1,178)=6.594, \mathrm{P}<0.05$. Experienced teachers show greater self-efficacy regarding student engagement than newly deployed teachers.

\section{Conclusion}

The results of the study have shown learning activities were poorly practice by teachers during CPD training. It also entails that what has been aspired to happen in classroom by lunching the CPD program not achieved and remained ambitious. In other words the as we live in the era of knowledge explosion there is a need for teachers to continually improve their knowledge and skill through CPD. It is hoped this can lead to better classroom achievement. However the results of the study have shown that very little of it was obtained both at school and classroom level.

This indicates that CPD program is adversely affected by, teachers' related factors such as their experience, level of education, their selfefficacy and perception of CPD.

\section{Recommendations}

Based on the findings and the conclusion drawn the following recommendation were forwarded.

Beliefs teachers hold influence their perceptions and judgment in which in turn affects their behavior both in and outside the classroom. Therefore teachers need to be provided with orientation or further awareness building training before they are involved in CPD training.

There is a need to inculcate in teachers belief that involvement in $\mathrm{CPD}$ is one of the major professional obligation and should be linked to career structure.

As teacher self-initiation and self-determination to attend and actively involved in CPD program appears to the key factors teachers should not rely solely on CPD course provided by external body, rather they need to take personal initiatives and commitment for the CPD 
Citation: Tareke G, Tefera B (2018) The Status and Psychological Challenges of Implementation of Continuous Professional Development/CPD/ in Primary Schools of South Wollo Zone. J Mass Communicat Journalism 8: 373. doi: 10.4172/2165-7912.1000373

Page 9 of 9

\begin{tabular}{|c|c|c|c|c|}
\hline Source of variation & Sum of Squares & df & Mean Square & $\mathbf{F}$ \\
\hline School type & 0.166 & 1 & 0.166 & 0.051 \\
\hline Experience & 21.624 & 1 & 21.624 & $6.594^{*}$ \\
\hline Gender & 1.639 & 1 & 1.639 & 0.500 \\
\hline School type $\times$ Experience & 0.897 & 1 & 0.897 & 0.274 \\
\hline School type $\times$ gender & 3.794 & 1 & 3.794 & 1.157 \\
\hline Experience $\times$ gender & 0.005 & 1 & 0.005 & 0.002 \\
\hline School type $\times$ Experience $\times$ gender & 2.266 & 1 & 2.266 & 0.691 \\
\hline Error & 583.704 & 178 & 3.279 & \\
\hline Total & 77104.000 & 186 & & \\
\hline
\end{tabular}

${ }^{*} \mathrm{P}<0.05$

Table 15: Summary of ANOVA: The Significance of Mean Difference of Teachers' Self-efficacy Regarding Student Engagement

program. Therefore, CPD needs should be needs that are identified at school level by the teacher themselves.

The CPD program should be carried out to fill the gap in teachers' knowledge and skills. It should not be a matter of repetition what teachers already known. It also should give emphasis to subject (content) matter knowledge.

Newly deployed teachers particularly those who graduated from colleges should receive training on basic teaching skills, such as, classroom management, assessment techniques and lesson planning as a basic requirement before they engaged in the actual CPD program.

Finally need assessment, planning, implementation; follow up and evaluation which operates in cyclic manner should be taken in account for better CPD implementation.

\section{References}

1. Tschannen-Moran H, Woolflok H (2002) The Differential Antecedents of Self Efficacy Beliefs of Novice and Experienced Teachers htth://www.renet.wm.edu. Retrieved on January 22, 2012.

2. Peery, Angela B (2004) Deep Change: Professional Development the Inside Out. USA: Littlefield Publishing Group, Inc.

3. Villegas- Reimers E (2003) Teachers Professional Development: An International Review of Literature. International Institute for Educational Planning, Paris: UNESCO.

4. Leu E (2004) The Patterns and Purposes of Localized Teacher Professional Development Programs. Academy for Educational Development.

5. Moon B, Butcher, J and Bird E (2000) Leading Professional Development in Education. London: RoutledgeFlamer.

6. Coto M (2010) A Community of Practice Approach to Facilitate University Teacher Professional Development in ICT and Project Based Problem Pedagogy. Denmark Aalborg University. (Un- published Thesis)

7. Anga E (2009) The status of Continuous Professional Development (CPD) Program in Government School, Unpublished M.A Thesis, Addis Ababa University.

8. Naru Sied (2005). Practice and Problems of School- Based Continuing
Professional Development of Teachers of Secondary School: the Case of Central Zone Tigray. Unpublished M.A Thesis, Addis Ababa University, Addis Ababa.

9. Alemayehu G (2010) Practice and Challenges of Continuous professional Development in Secondary Schools of Bale Zone. Unpublished M.A Thesis, Addis Ababa University, Addis Ababa.

10. Yhunie M (2008) Practice and Challenges of Continuous professional Development in Primary Schools of West Gojam Zone. Unpublished M.A Thesis, Addis Ababa University, Addis Ababa.

11. Hailu E, Jabessa F (2010) Teachers' Perceptions of School Based Continuous Professional Development (SBCPD) in Jimma Zone Selected Schools. Ethiopian Journal of Education and Science 5: 59-76.

12. Feiman-Nemser S (2002) Form Preparation to Practice: Designing a Continuum to Strengthen and Sustain Teaching. Teachers College Record 105: 10131055.

13. Harwell S (2002) Teacher-Centered Professional Development: It is Not an Event, It is a Process. http:www.org/uploadfile.Retrieved January 2, 2012.

14. Lemke C (2010) Professional Development Ensuring a Return on Your Investment. Commissioned by Intel.

15. Mundry S (2005) What Experience Has Thought Us about professional development: Facilitating Mathematics and Science Reform.

16. Bransfords S (1999) Managing Professional Development in School. London: St.Edmurdsburg Press.

17. Maldonao L (2002) Effective Professional Development: Finding from Research. K-12 professional development, College Board Advanced Placement Program.

18. Day C (1999) Developing Teachers; The challenges of lifelong Learning. New York: RutledgeFalmer

19. Craft A (2004) Continuing Professional Development: a practical Guide for Teacher and School. New York: RoutledgeFalmer.

20. Smith C and Hofer J (2003) The Characteristics and Concerns of Adult Basic Education. Boston: National Center for the Study of Adult Learning and Literacy.

21. Bennet J, Braund M, Lubben F (2010) The Impact of Targeted Continuing Professional Development (CPD) on Teachers' Professional Practice in Science. Report Commissioned by the DFCS through the National Science Learning Center. York: University of York, Department of Educational Studies. 